\author{
R. Dikkers \\ T. P. Willems \\ L. H. Piers \\ G. J. de Jonge \\ R. A. Tio \\ H. J. van der Zaag-Loonen \\ P. M. A. van Ooijen \\ F. Zijlstra \\ M. Oudkerk
}

\title{
Coronary revascularization treatment based on dual-source computed tomography
}

Received: 25 July 2007

Revised: 7 February 2008

Accepted: 23 February 2008

Published online: 20 May 2008

(C) The Author(s) 2008

R. Dikkers $(\bowtie) \cdot$ T. P. Willems •

G. J. de Jonge .

H. J. van der Zaag-Loonen .

P. M. A. van Ooijen - M. Oudkerk

Department of Radiology,

University Medical Center Groningen,

University of Groningen,

Hanzeplein 1,

P.O. Box 30.001, 9700 RB

Groningen, The Netherlands

e-mail: r.dikkers@rad.umcg.nl

Tel.: +31-5036-14260

Fax: +31-5036-11798

L. H. Piers · R. A. Tio - F. Zijlstra

Department of Cardiology,

University Medical Center Groningen,

University of Groningen,

Groningen, The Netherlands

\begin{abstract}
Therapy advice based on dual-source computed tomography (DSCT) in comparison with coronary angiography (CAG) was investigated and the results evaluated after 1-year follow-up. Thirty-three consecutive patients (mean age 61.9 years) underwent DSCT and CAG and were evaluated independently. In an expert reading (the "gold standard"), CAG and DSCT examinations were evaluated simultaneously by an experienced radiologist and cardiologist. Based on the presence of significant stenosis and current guidelines, therapy advice was given by all readers blinded from the results of other readings and clinical information. Patients were treated based on a multidisciplinary team evaluation including all clinical information. In comparison with the gold standard, CAG had a higher
\end{abstract}

specificity (91\%) and positive predictive value (PPV) (95\%) compared with DSCT $(82 \%$ and $91 \%$, respectively). DSCT had a higher sensitivity (96\%) and negative predictive value (NPV) $(89 \%)$ compared with CAG (91\% and $83 \%$, respectively). The DSCT-based therapy advice did not lead to any patient being denied the revascularization they needed according to the multidisciplinary team evaluation. During follow-up, two patients needed additional revascularization. The high NPV for DSCT for revascularization assessment indicates that DSCT could be safely used to select patients benefiting from medical therapy only.

Keywords Computed tomography . Coronary artery disease .

Therapy advice

\section{Introduction}

High diagnostic accuracy of multi-detector computed tomography (MDCT) has been assessed in comparison with coronary angiography (CAG) (the "gold standard") for coronary artery stenosis by several recent studies [1-6].

The recently introduced dual-source CT (DSCT) has improved temporal resolution, which results in a high image quality of the distal segments even in patients with a heart rate above 80 beats per minute (bpm) [7-9]. Now, in several studies, the potential diagnostic impact of coronary CT angiography has been investigated to see whether it can surpass diagnostic CAG in the near future $[10,11]$. This development has important implications for patients with coronary artery disease, especially for the assessment of a correct treatment strategy, which is currently based on CAG findings [12-14]. The therapy can be percutaneous coronary intervention (PCI), coronary artery bypass graft $(\mathrm{CABG})$ surgery or medical management. However, the choice of therapy choice depends not only on the number of diseased coronary arteries but, even more importantly, on which coronary artery is diseased [12-14]. For example, a left main stenosis will be treated with CABG surgery but a single stenosis in the proximal right coronary artery will be sufficiently treated with PCI. Distal branches smaller than $2 \mathrm{~mm}$ in diameter will in general not be treated with stent placement or CABG surgery, since they are too small. Therefore, the diagnostic accuracy of stenosis detection on a segment level does not reflect the clinical impact of DSCT for treatment advice. 
The purpose of this study was to investigate the potential of therapy advice based on DSCT in comparison with CAG and to evaluate the results after 1-year follow-up.

\section{Materials and methods}

\section{Patients}

Consecutive patients scheduled for diagnostic CAG or elective PCI were included in this study from April 24th 2006 till September 1st 2006. The study protocol was approved by the ethics committee of the hospital and informed consent was obtained from all patients before the examinations. Exclusion criteria were: previous reaction to iodine contrast media, hyperthyroidism, severe renal insufficiency (creatine levels $>120 \mu \mathrm{mol} / \mathrm{l}$ ), atrial fibrillation, unstable clinical condition, inability to follow breathhold commands, previous coronary intervention or bypass surgery. All patients underwent both DSCT and CAG.

\section{DSCT}

Coronary CT angiography was performed on a DSCT system (Somatom Definition, Siemens Medical Systems, Forchheim, Germany). All patients received nitroglycerine spray (Nitrolingual pump spray, $0.4 \mathrm{mg} /$ dose, PohlBoskamp) but no additional beta-blockers were administrated in preparation of the scan. Mean heart rate during CT was $60( \pm 13) \mathrm{bpm}$, range $40-90 \mathrm{bpm}$. A retrospective ECG-triggered CT data acquisition was made with a gantry rotation time of $0.33 \mathrm{~s}$, a tube current of $412 \mathrm{mAs} /$ rotation and a tube voltage of $120 \mathrm{kV}$. ECG pulsing window was set to $20-70 \%$ of the RR-interval and was used for all patients. The pitch was automatically adapted to the heart rate (range $0.2-0.5$ ). Direction of data acquisition was craniocaudal starting above the coronary ostia and ending at the diaphragm below all cardiac structures. For contrast enhancement, $73 \mathrm{ml}$ of non-ionic contrast agent (Iomeprol $400 \mathrm{mg} \mathrm{I} / \mathrm{ml}$; Iomeron 400, Bracco, Italy) was injected in an antecubital vein followed by a saline bolus using bolus tracking with a region of interest (ROI) in the descending aorta for timing.

Images were reconstructed with $0.6-\mathrm{mm}$ slice thickness and $0.4-\mathrm{mm}$ increment after selecting the cardiac phase for each coronary artery showing the least apparent motion. The calculated mean effective radiation dose based on the used scan protocol was $7.3 \mathrm{mSv}$ [15].

\section{CAG procedure}

CAG, with or without stent placement, was performed using the standard procedure via the femoral or radial artery. All cine-runs were stored on a disk for off-line evaluation.
Treatment

Patients were treated by cardiologists not involved in the current study. Treatment was based on the current guidelines using the CAG for stenosis evaluation [12-14]. Furthermore, the patient's physical condition and medical history were also taken into account during a multidisciplinary meeting with at least a cardiologist, an interventional cardiologist and a thoracic surgeon, deciding on the best treatment for every individual patient. All patients were followed for at least 1 year for the occurrence of recurrent angina, cardiac events or mortality.

\section{Data evaluation}

\section{Readers}

Both a senior reader (2 years' experience for CT CAG and at least 5 years' experience for $\mathrm{CAG}$ ) and a junior reader (with little experience for CT CAG and CAG) evaluated the images. All readers were blinded from the results of other readers and from patient's medical history and clinical follow-up. A significant stenosis was defined as an area stenosis of $>75 \%$ on DSCT using dedicated cardiac software (Circulation, Siemens, Forchheim, Germany). A significant stenosis on CAG was defined as a diameter stenosis of $>50 \%$ by visual assessment.

Based on the evaluation of the images, therapy advice was given. In a second reading, the junior and senior reader of DSCT and CAG, respectively, read the DSCT and CAG examinations in consensus.

The therapy advice of the different readers was compared with the actual treatment given to the patients based on the multidisciplinary meeting and compared with an expert reading. The expert reading was performed blinded from the other readings. In a joint session, an experienced interventional cardiologist and an experienced cardiac radiologist evaluated simultaneously the CAG and DSCT examination. Advice about therapy was given based on the guidelines after consensus was reached between both readers [12-14]. The expert reading was used as the gold standard because it was performed blinded from the patient's medical history and clinical follow-up comparable with the other readers.

\section{Therapy advice}

Therapy advice could consist of medical management, PCI and $\mathrm{CABG}$, and was based on the recent guidelines of the American College of Cardiology and American Heart Association and European Society of Cardiology [12-14]. Medical management was advised in case of non-significant stenosis. CABG was advised when there was (1) a significant obstruction of the left main, (2) three-vessel disease (i.e. three 
different coronary vessels with a significant stenosis), or (3) two-vessel disease (i.e. two different coronary vessels with a significant stenosis) including a proximal LAD stenosis. PCI was advised in all other cases of single and two vessel disease.

Afterwards, patients were grouped for diagnostic accuracy assessment in needing revascularization or not needing revascularization based on the given therapy advice.

\section{Follow-up}

All patients were followed for at least 1 year. Patients were seen at least once by the cardiologist at the out-patient clinic after the CAG procedure. Medical records were evaluated for any adverse cardiac events of mortality.

\section{Statistical analysis}

Non-assessable segments were considered free of any stenosis for all readers and for both modalities. Kappa values were calculated for agreement in therapy advice. Kappa values between 0 and 0.40 were considered poor, $0.41-0.60$ moderate, $0.61-0.80$ good and $>0.81$ excellent [16]. For the diagnostic accuracy of predicting revascularization, the sensitivity, specificity, positive predictive value (PPV) and negative predictive value (NPV) were calculated. Continuous data are expressed as mean \pm standard deviation $( \pm \mathrm{SD})$. The median and range were given for skewed data.

\section{Results}

Thirty-three consecutive patients were included in the study with a mean age 61.9 years and a male predominance $(82 \%)$. Table 1 lists the prevalence of risk factors in the included patients. All 33 patients could be evaluated on both modalities and by all observers. Based on the

Table 1 Patient characteristics

\begin{tabular}{ll}
\hline & Number of patients $(\%)$ \\
\hline Mean age $( \pm$ SD) & $61.9( \pm 10.3)$ \\
Male & $27(82)$ \\
Risk factors: & $11(33)$ \\
Smoking & $11(33)$ \\
Hypercholesterolemia & $10(30)$ \\
Familial predisposition & $9(27)$ \\
Hypertension & $6(18)$ \\
Diabetes mellitus &
\end{tabular}

multidisciplinary team evaluation patients were treated as follows; 17 patients underwent medical management, ten patients underwent $\mathrm{PCI}$, and six patients underwent $\mathrm{CABG}$ surgery.

Treatment based on the multidisciplinary team evaluation

The overall agreement for therapy advice of the individual readers compared with the multidisciplinary team evaluation was moderate to good (Table 2). The agreement of the expert reading compared with the actual treatment was moderate (kappa 0.59).

The discrepancies in therapy advice between the expert reading and the treatment based on the multidisciplinary team evaluation are explained as follows: two patients who needed PCI treatment based on CAG and DSCT during the expert reading were treated with medical therapy since collateral flow was present. The presence of collateral flow was not evaluated by the CAG observers and could not be seen on DSCT. Four patients needing CABG surgery according to the expert reading were treated with medical therapy (two patients) and with PCI (the two other patients). No revascularization options in the first two mentioned patients were available and a limited physical condition in the latter two mentioned patients was present.

Three false-positive findings of the expert reading could not be explained by the lack of clinical information. Two of three patients were incorrectly diagnosed as having a significant stenosis in the proximal left circumflex artery (LCx) and distal LCx, respectively. One other falsepositive finding of a significant stenosis in the proximal left anterior descending artery (LAD) resulted in an incorrect $\mathrm{CABG}$ advice. This patient was treated with PCI instead. However, 5 months later CABG surgery was performed since symptoms persisted (patient 3). This last finding could, therefore, be seen as a false negative finding of the multidisciplinary team evaluation rather than a falsepositive finding by the expert reading. No false-negative findings were observed for the expert reading in comparison with the treatment based on the multidisciplinary team evaluation. There were also no false-negative findings for the senior reader of CAG and all readers of DSCT in comparison with the treatment based on the multidisciplinary team evaluation.

Therapy advice based on the expert reading (gold standard)

The agreement of CAG and DSCT compared with the expert reading was good to excellent (range 0.65-0.82) (Table 2). Senior readers showed higher agreement with the expert reading compared with the junior reader of the same techique $(0.67$ vs 0.82 and 0.65 vs 0.79 , for $\mathrm{CAG}$ and 
Table 2 Agreement in therapy advice $(95 \%$ CI $95 \%$ confidence interval, junior junior reader, senior senior reader, consensus consensus reading between junior and senior reader, expert expert reading during a joint reading of CAG and DSCT, multidisciplinary team therapy advice based on the multidisciplinary team evaluation)

\begin{tabular}{llllll}
\hline Technique & Reader & Expert & & \multicolumn{2}{l}{ Multidisciplinary team } \\
\hline \multirow{2}{*}{ CAG } & & Kappa value & $95 \%$ CI & Kappa value & $95 \%$ CI \\
& & & & & \\
& Junior & 0.67 & $0.48-0.87$ & 0.54 & $0.30-0.77$ \\
& Senior & 0.82 & $0.70-0.97$ & 0.68 & $0.49-0.88$ \\
DSCT & Consensus & 0.76 & $0.59-0.92$ & 0.65 & $0.46-0.83$ \\
& & & & & \\
& Junior & 0.65 & $0.46-95$ & 0.47 & $0.26-0.70$ \\
& Senior & 0.79 & $0.63-95$ & 0.59 & $0.36-0.81$ \\
CAG + DSCT & Consensus & 0.79 & $0.63-95$ & 0.59 & $0.37-0.81$ \\
& & & & & $0.39-0.82$ \\
\hline
\end{tabular}

DSCT, respectively). The difference in kappa value between the different techniques is less in comparison with the expert reading than in comparison with the multidisciplinary team evaluation. Figure 1 shows a patient in which both CAG and DSCT detected a significant LAD stenosis and both advised PCI based on this finding.

\section{Revascularization advice}

When looking at the advice for revascularization or no revascularization (Table 3 ) the same pattern was observed: low levels of agreement for all readers including the expert reading in comparison with the multidisciplinary team evaluation; higher levels of agreement for the senior reader compared with the junior reader of the same technique; and less difference in the level of agreement between techniques in comparison with the expert reading than compared with the multidisciplinary team evaluation.

\section{Diagnostic accuracy}

Diagnostic accuracy for the assessment of the need for revascularization was also calculated in comparison with the multidisciplinary team evaluation (Table 4), in comparison with the expert reading (gold standard) (Table 5), and for DSCT in comparison with CAG (Table 6). In general, the senior reader had a higher accuracy compared with the junior reader.

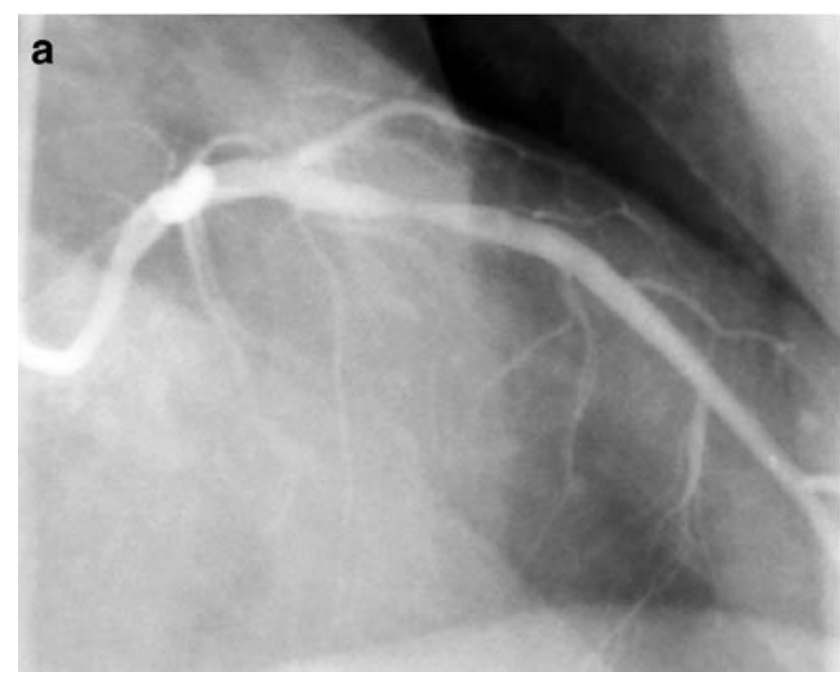

Fig. 1 a CAG image of a significant stenosis in the LAD artery and b the corresponding 3D volume-rendering image and c curved multi-planar reconstruction (MPR) image of DSCT of the same
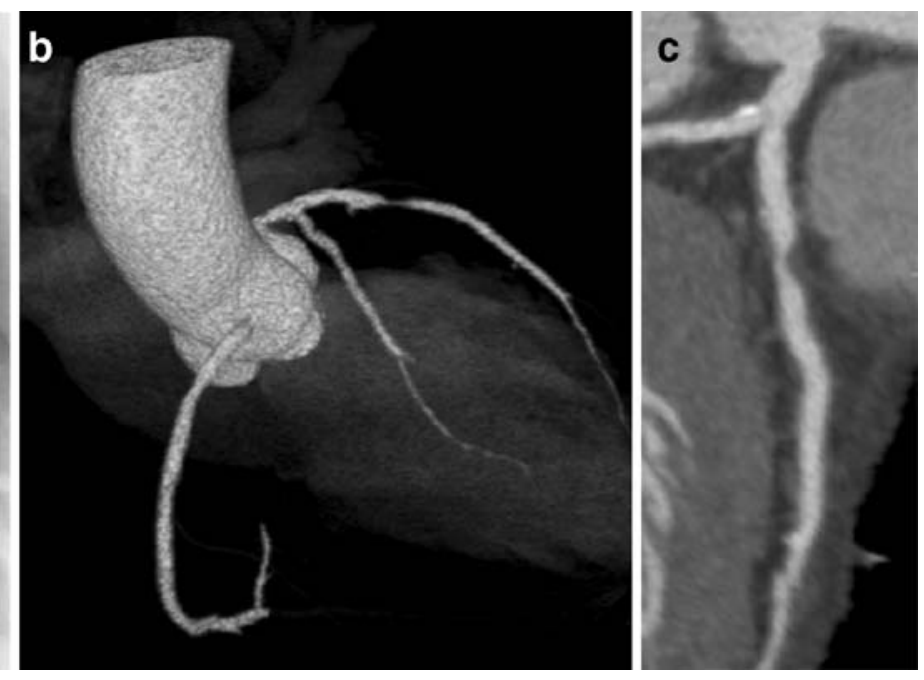

patient. Based on these images, revascularisation (PCI) was advised by both CAG and DSCT 
Table 3 Agreement in revascularization advice

\begin{tabular}{llllll}
\hline Technique & Reader & Expert & & \multicolumn{2}{l}{ Multidisciplinary team } \\
\hline \multirow{4}{*}{ CAG } & & Kappa value & $95 \%$ CI & Kappa value & $95 \%$ CI \\
& & & & & \\
& Junior & 0.80 & $0.59-1.00$ & 0.58 & $0.32-0.78$ \\
& Senior & 0.87 & $0.70-1.00$ & 0.76 & $0.54-0.97$ \\
DSCT & Consensus & 0.79 & $0.57-1.00$ & 0.80 & $0.59-1.00$ \\
& & & & & \\
& Junior & 0.63 & $0.35-0.92$ & 0.46 & $0.21-0.72$ \\
& Senior & 0.71 & $0.46-0.97$ & 0.52 & $0.27-0.78$ \\
CAG + DSCT & Consensus & 0.74 & $0.46-1.00$ & 0.58 & $0.33-0.83$ \\
& & & & & $0.40-0.88$ \\
\hline
\end{tabular}

Diagnostic accuracy compared

with the multidisciplinary team evaluation

Compared with DSCT, CAG demonstrated a higher specificity $(65 \%, 77 \%$, and $71 \%$ vs $47 \%, 53 \%$, and $59 \%$ for the junior, senior and consensus reading of CAG and DSCT, respectively) and PPV $(71 \%, 80 \%$, and $76 \%$ vs. $64 \%, 67 \%$, and $70 \%$ for the junior, senior and consensus reading of CAG and DSCT, respectively) (Table 4). The junior reader of DSCT showed a higher sensitivity and negative predictive value compared with the junior reader of CAG $(100 \%$ and $100 \%$ vs $94 \%$ and $92 \%$ for DSCT and CAG, respectively).

\section{Diagnostic accuracy compared with the expert reading (gold standard)}

CAG showed a higher specificity and PPV than DSCT for all readers in comparison with the expert reading (Table 5). DSCT showed a higher sensitivity and NPV than CAG for all readers in comparison with the expert reading. Large confidence intervals were seen for all observers and both modalities with a large overlap.

\section{Diagnostic accuracy of DSCT compared with CAG}

In a comparison between DSCT and CAG, a higher accuracy is seen for the senior reader (Table 6). In the consensus reading, a moderate specificity, PPV and NPV is found for DSCT in comparison with CAG, with large confidence intervals. Sensitivity of DSCT compared with CAG is good with $95 \%$. Overall diagnostic accuracy was $88 \%$. In three patients DSCT advised a PCI instead of medical treatment advised by CAG. In one of these patients the expert reading also advised a PCI. In one patient, DSCT advised medical treatment where CAG advised PCI. This patient, however, was treated with medical treatment only based on the multidisciplinary team evaluation. The expert reading also advised medical treatment in this particular patient.

Table 4 Diagnostic accuracy of revascularization advice based on CAG and DSCT compared with the multidisciplinary team evaluation. (Numbers are percentages)

\begin{tabular}{|c|c|c|c|c|c|c|c|c|c|}
\hline \multicolumn{10}{|c|}{ Multidisciplinary team } \\
\hline Technique & Reader & Sensitivity & $95 \% C I$ & Specificity & $95 \% C I$ & $P P V$ & $95 \% C I$ & $N P V$ & $95 \% C I$ \\
\hline \multicolumn{10}{|l|}{ CAG } \\
\hline & Junior & 94 & $82-100$ & 65 & $42-87$ & 71 & $52-91$ & 92 & $76-100$ \\
\hline & Senior & 100 & $100-100$ & 77 & $56-97$ & 80 & $63-98$ & 100 & $100-100$ \\
\hline & Consensus & 100 & $100-100$ & 71 & $49-92$ & 76 & $58-94$ & 100 & $100-100$ \\
\hline \multicolumn{10}{|l|}{ DSCT } \\
\hline & Junior & 100 & $100-100$ & 47 & $23-71$ & 64 & $45-83$ & 100 & $100-100$ \\
\hline & Senior & 100 & $100-100$ & 53 & $29-77$ & 67 & $47-86$ & 100 & $100-100$ \\
\hline & Consensus & 100 & $100-100$ & 59 & $35-82$ & 70 & $51-88$ & 100 & $100-100$ \\
\hline \multicolumn{10}{|c|}{$\mathrm{CAG}+\mathrm{DSCT}$} \\
\hline & Expert & 100 & $100-100$ & 65 & $42-87$ & 73 & $54-91$ & 100 & $100-100$ \\
\hline
\end{tabular}


Table 5 Diagnostic accuracy of revascularization advice based on CAG and DSCT compared with the expert reading. (Numbers are percentages)

\begin{tabular}{|c|c|c|c|c|c|c|c|c|c|}
\hline \multicolumn{10}{|c|}{ Expert reading } \\
\hline Technique & Reader & Sens. & $95 \% C I$ & Spec. & $95 \% C I$ & $P P V$ & $95 \% C I$ & $N P V$ & $95 \% C I$ \\
\hline \multicolumn{10}{|l|}{ CAG } \\
\hline & Junior & 91 & $79-100$ & 91 & $74-100$ & 95 & $86-100$ & 83 & $62-100$ \\
\hline & Senior & 91 & $79-100$ & 100 & $100-100$ & 100 & $100-100$ & 85 & $65-100$ \\
\hline & Consensus & 91 & $79-100$ & 91 & $74-100$ & 95 & $86-100$ & 83 & $62-100$ \\
\hline \multicolumn{10}{|l|}{ DSCT } \\
\hline & Junior & 96 & $87-100$ & 64 & $35-92$ & 84 & $70-98$ & 88 & $65-100$ \\
\hline & Senior & 96 & $87-100$ & 73 & $46-99$ & 88 & $74-100$ & 89 & $68-100$ \\
\hline & Consensus & 96 & $87-100$ & 82 & $59-100$ & 91 & $80-100$ & 90 & $71-100$ \\
\hline
\end{tabular}

\section{Inter-observer agreement}

The inter-observer agreement for therapy advice between the junior and senior reader is 0.74 (95\% CI: $0.54-0.93$ ) for CAG and 0.63 (95\% CI: $0.41-0.85$ ) for DSCT. The interobserver agreement for revascularization between the junior and senior reader is 0.81 (95\% CI: $0.60-1.00)$ for CAG and 0.60 (95\% CI: $0.29-0.92$ ) for DSCT.

\section{Follow-up}

After a 1-year follow up (mean 1.3 year \pm 1 month), 28 patients had no cardiac events and were without symptoms. Three patients have died of non-cardiac-related causes. Two patients received additional revascularization after their initial treatment.

Patient 1 with atypical chest pain and non-significant coronary artery stenosis on the initial diagnostic CAG was treated with medical therapy (Fig. 2). After 7 months this patient presented at the emergency department with a STelevated myocardial infarction and PCI with stent placement was performed. The CAG at that time showed a stenosis of $90 \%$ in the proximal right coronary artery (RCA) (Fig. 2b). Also retrospectively, the initial diagnostic CAG did not show any signs of stenosis or wall irregularities (Fig. 2a). On DSCT, a mixed plaque consisting of calcification and soft plaque was seen in the proximal RCA by both DSCT observers but did not result in an area stenosis $>75 \%$ (Fig. 3).

Patient 2 initially underwent PCI of the proximal LCx. Five months later, $\mathrm{CABG}$ surgery was performed because of invalidating angina pectoris caused by a significant stenosis in the LAD. This stenosis was earlier mistaken for an artefact caused by side branches both during clinical evaluation as during the CAG readings (Fig. 4). This stenosis in the proximal LAD was seen on DSCT as an area stenosis of $>75 \%$ by all DSCT observers (Fig. 5). Based on the DSCT findings revascularization would have been indicated.

Table 6 Diagnostic accuracy of revascularization advice CAG vs DSCT. (Numbers are percentages)

\begin{tabular}{|c|c|c|c|c|c|c|c|c|}
\hline \multicolumn{9}{|l|}{ CAG junior } \\
\hline DSCT & Sens. & $95 \% C I$ & Spec & $95 \% C I$ & $P P V$ & $95 \% C I$ & $N P V$ & $95 \% C I$ \\
\hline Junior & 95 & $86-100$ & 58 & $30-86$ & 80 & 64-96 & 88 & $65-100$ \\
\hline Senior & 91 & $78-100$ & 58 & $30-86$ & 79 & $63-95$ & 78 & $51-100$ \\
\hline \multicolumn{9}{|l|}{ CAG senior } \\
\hline & Sens. & $95 \% C I$ & Spec. & $95 \% C I$ & $P P V$ & $95 \% C I$ & $N P V$ & $95 \% C I$ \\
\hline Junior & 100 & $100-100$ & 62 & $35-88$ & 80 & 64-96 & 100 & $100-100$ \\
\hline Senior & 100 & $100-100$ & 69 & $44-94$ & 83 & $68-98$ & 100 & $100-100$ \\
\hline \multicolumn{9}{|c|}{ CAG consensus } \\
\hline & Sens. & $95 \% C I$ & Spec. & $95 \% C I$ & $P P V$ & $95 \% C I$ & $N P V$ & $95 \% C I$ \\
\hline Consensus & 95 & $86-100$ & 75 & $51-100$ & 87 & $73-100$ & 90 & $71-100$ \\
\hline
\end{tabular}



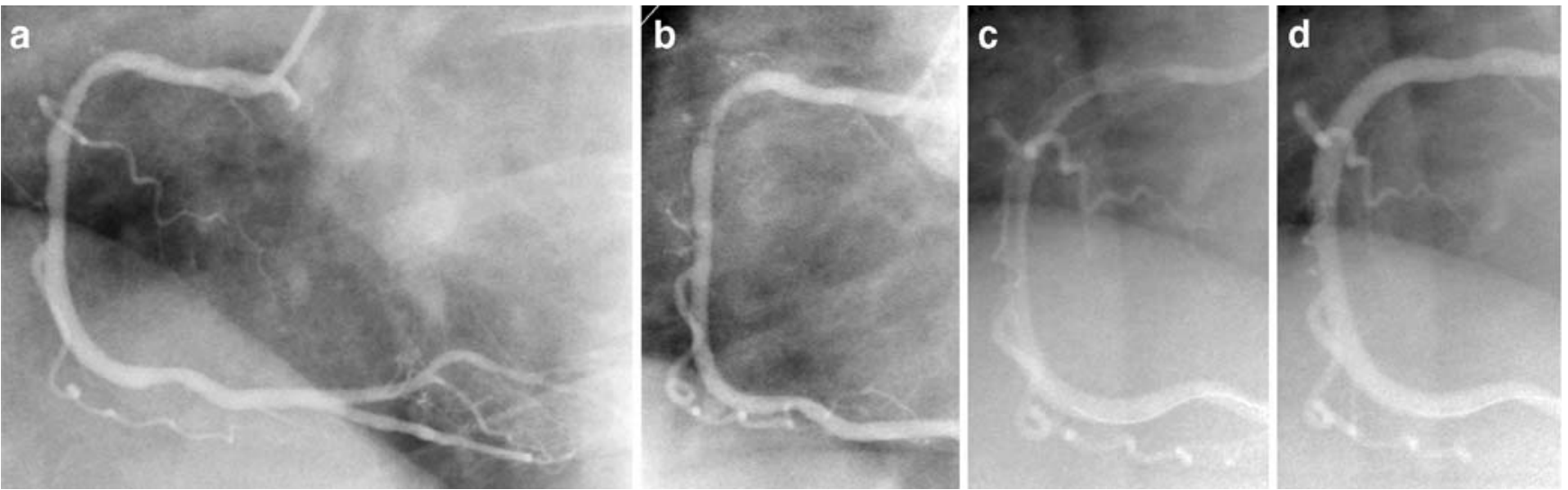

Fig. 2 CAG images of the RCA of patient 2. a CAG at initial presentation shows no significant stenosis. b The same patient presented with ST-elevated myocardial infarction 7 months later. CAG shows a proximal RCA stenosis and an occlusion of the right

\section{Discussion}

This study evaluated the potential of a DSCT-based therapy advice using the current cardiac intervention guidelines [12-14]. DSCT and CAG data were compared with the same reference standards, namely the expert reading and the multidisciplinary team evaluation. Based on clinical information, six out of 33 patients $(18 \%)$ received a different treatment other then according to the guidelines would be appropriate. These deviations from the guidelines resulted in a low level of agreement for both modalities and all observers compared with the treatment based on the multidisciplinary team evaluation.
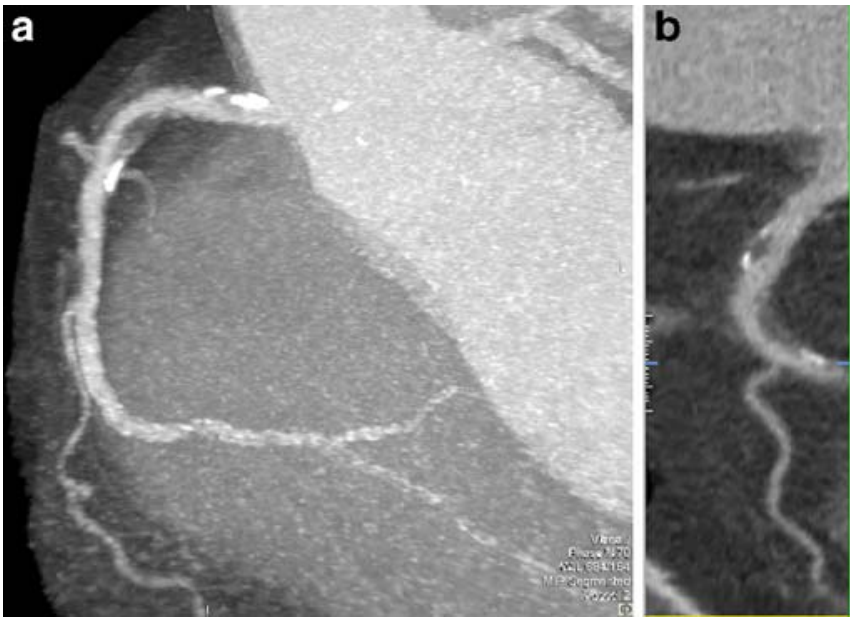

Fig. 3 a DSCT image of the RCA of patient 2 at initial presentation shows a mixed plaque in the proximal RCA, resulting in a stenosis of $50 \%$. A second, mainly calcified plaque is present near the ostium. b DSCT image shows the mixed plaque in more detail together with the right ventricular branch of the RCA ventricular branch. c CAG image of the stent in the proximal RCA. A guide-wire is visible distal in the RCA. d Result after PCI with stent placement showing the right ventricular branch filling with contrast again

In comparison with the expert reading (gold standard) which was also performed blinded from clinical information both techniques performed equally well. Since the patient group was small and the $95 \%$ confidence interval for both techniques was large, we concluded that no clinical difference in agreement was seen. The agreement between DSCT and the expert reading on therapy advice (kappa value 0.79 ) is in accordance with earlier findings of Dorgelo et al. [17], who also corrected for changes in treatment strategy based on clinical information (kappa 0.76).

For revascularization advice, DSCT showed a lower specificity and PPV than CAG compared with the gold standard. From the literature it is known that the specificity

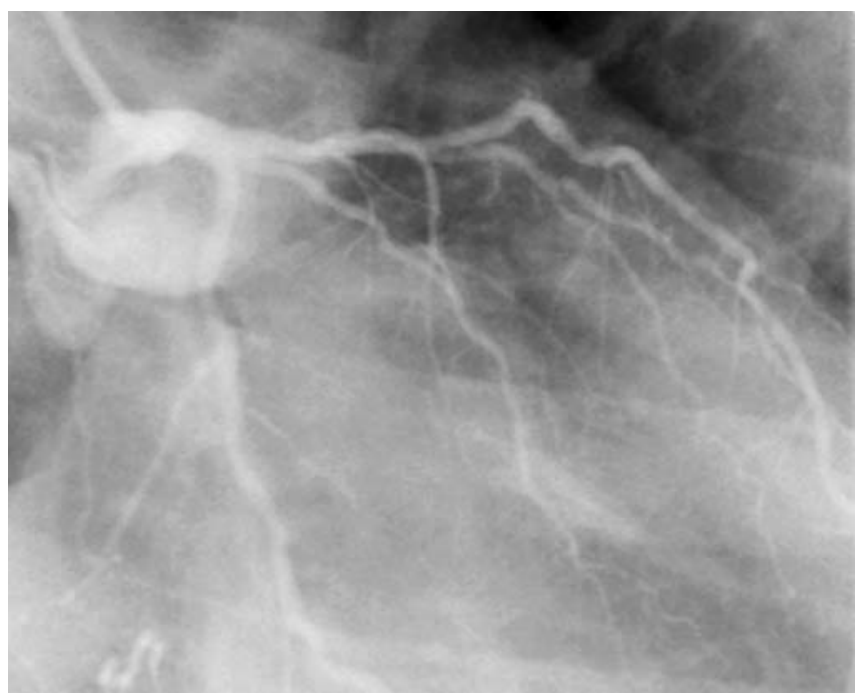

Fig. 4 CAG of patient 3 shows no significant stenosis of the LAD artery 
Fig. 5 DSCT images of patient 3. a Curved MPR of the LAD artery and an axial image perpendicular to the vessel (insert) shows the significant lumen stenosis in the proximal LAD (line) caused by a calcified plaque. The remaining lumen is indicated. b Three-dimensional volume-rendered image shows from left to right the RCA, $\mathrm{LAD}$, the first diagonal branch and the LCx artery with multiple calcified plaques
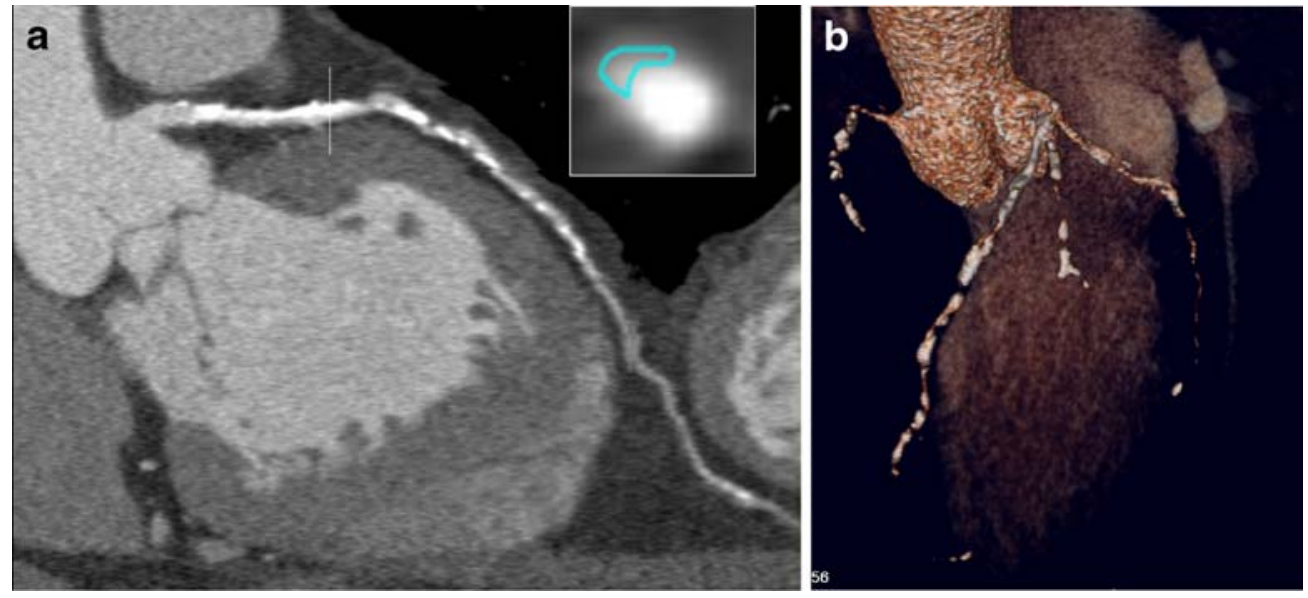

and PPV of CT CAG remains low even for the more recent CT systems [4, 6, 18, 19]. This is caused by the overestimation of stenosis severity by CT angiography in the presence of severe calcification and stents [2, 20,21]. Motion artefacts due to irregular heart rate and breathing artefacts can also result in image quality degradation, and result in a lower diagnostic accuracy [20].

On the other hand, the absence of coronary plaques on CT angiography has proven to safely exclude the presence of coronary artery disease and result in a high NPV $[5,11]$. This study indicates that DSCT can be used to select patients' not needing revascularization, since no patient receiving revascularization based on the multidisciplinary team evaluation was missed. These patients potentially do not need to undergo invasive CAG in the future and can be safely sent home with extensive medical management. In our follow-up period of at least 1 year, only two patients needed additional revascularization. Based on the DSCT, patient 2 would have been senT for CABG and would have been spared the initial PCI procedure. Patient 1 would have been treated with medical treatment based on the DSCT finding. This patient is, however, one example of the difficult cases in which there might be doubt about the best treatment approach. This patient was treated according to the current guidelines. From pathological studies it is known that mixed plaques seen on DSCT with a large soft plaque component are prone to rupture [22-24]. Current guidelines still do not advise protective stenting of these lesions seen on DSCT in the lack of clinical evidence. It remains striking, however, how these large plaques are completely missed by $\mathrm{CAG}$, even retrospectively.

The improved temporal resolution of DSCT has shown to result in high diagnostic accuracy of stenosis detection in patients with heart rates above $80 \mathrm{bpm}[6,9]$. Further improvement of the spatial resolution for CT angiography is needed to improve image quality and more importantly diagnostic accuracy in patients with severe calcifications and stents.
The major limitation of CT angiography is still the fact that it provides only anatomical and some morphological information about coronary arteries and plaques. It does not give functional information about flow especially retrograde flow and the presence of ischemia. Therefore, the clinical implications of stenosis severity and with that the decision for PCI or CABG based on DSCT findings can be limited.

\section{Limitations}

The number of patients included was rather small and patients generally had a relatively low heart rate. New studies including patients with higher heart rates and irregular heart rates are needed to establish the role of DSCT in these specific patient groups.

\section{Conclusion}

This study showing a high NPV for DSCT for revascularization assessment indicates that DSCT could be safely used to select patients benefiting from medical therapy only. In comparison with the expert reading (the gold standard), there is no clinical difference between the treatment prediction of CAG and DSCT. For both techniques experience is essential, however, for the most accurate diagnosis and treatment advice.

Acknowledgements The authors thank G.A.J. Jesserun for evaluating CAG data, R. Mulder for his technical support and E.J. $\mathrm{K}$. Noach for critically evaluating the manuscript.

Open Access This article is distributed under the terms of the Creative Commons Attribution Noncommercial License which permits any noncommercial use, distribution, and reproduction in any medium, provided the original author(s) and source are credited. 


\section{References}

1. Butler J, Shapiro M, Reiber J et al (2007) Extent and distribution of coronary artery disease: a comparative study of invasive versus noninvasive angiography with computed angiography. Am Heart J 153:378-384

2. Ehara M, Surmely JF, Kawai M et al (2006) Diagnostic accuracy of 64-slice computed tomography for detecting angiographically significant coronary artery stenosis in an unselected consecutive patient population: comparison with conventional invasive angiography. Circ J 70:564-571

3. Fine JJ, Hopkins CB, Ruff N, Newton FC (2006) Comparison of accuracy of 64-slice cardiovascular computed tomography with coronary angiography in patients with suspected coronary artery disease. Am J Cardiol 97:173174

4. Leschka S, Alkadhi H, Plass et al (2005) Accuracy of MSCT coronary angiography with 64-slice technology: first experience. Eur Heart J 26:14821487

5. Oncel D, Oncel G, Tastan A, Tamci B (2007) Detection of significant coronary artery stenosis with 64-section MDCT angiography. Eur J Radiol 62:394-405

6. Scheffel H, Alkadhi H, Plass et al (2006) Accuracy of dual-source CT coronary angiography: First experience in a high pre-test probability population without heart rate control. Eur Radiol 16:2739-2747

7. Achenbach S, Ropers D, Kuettner A et al (2006) Contrast-enhanced coronary artery visualization by dual-source computed tomography-initial experience. Eur J Radiol 57:331-335

8. Flohr TG, McCollough $\mathrm{CH}$, Bruder $\mathrm{H}$ et al (2006) First performance evaluation of a dual-source CT (DSCT) system. Eur Radiol 16:256-268
9. Johnson TR, Nikolaou K, Wintersperger BJ et al (2006) Dualsource CT cardiac imaging: initial experience. Eur Radiol 16:1409-1415

10. Mollet NR, Cademartiri F, Van Mieghem C et al (2007) Adjunctive value of CT coronary angiography in the diagnostic work-up of patients with typical angina pectoris. Eur Heart $\mathrm{J}$ 28:1872-1878

11. Pundziute G, Schuijf JD, Jukema JW et al (2007) Prognostic value of multislice computed tomography coronary angiography in patients with known or suspected coronary artery disease. $\mathrm{J}$ Am Coll Cardiol 49:62-70

12. Anderson JL, Adams CD, Antman EM et al (2007) ACC/AHA 2007 guidelines for the management of patients with unstable angina/non-ST-Elevation myocardial infarction: a report of the American College of Cardiology/ American Heart Association Task Force on Practice Guidelines (Writing Committee to Revise the 2002 Guidelines for the Management of Patients With Unstable Angina/NonST-Elevation Myocardial Infarction) developed in collaboration with the American College of Emergency Physicians, the Society for Cardiovascular Angiography and Interventions, and the Society of Thoracic Surgeons endorsed by the American Association of Cardiovascular and Pulmonary Rehabilitation and the Society for Academic Emergency Medicine. J Am Coll Cardiol 50:e1-e157

13. Bassand JP, Hamm CW, Ardissino D et al (2007) Guidelines for the diagnosis and treatment of non-ST-segment elevation acute coronary syndromes. The Task Force for the Diagnosis and Treatment of Non-ST-Segment Elevation Acute Coronary Syndromes of the European Society of Cardiology. Eur Heart J 28:1598-1660

14. Fox K, Garcia MA, Ardissino D et al (2006) Guidelines on the management of stable angina pectoris: executive summary: the Task Force on the Management of Stable Angina Pectoris of the European Society of Cardiology. Eur Heart J 27:1341-1381
15. Gerber TC, Kuzo RS, Morin RL (2005) Techniques and parameters for estimating radiation exposure and dose in cardiac computed tomography. Int $\mathrm{J}$ Cardiovase Imaging 21:165-176

16. Landis JR, Koch GG (1977) The measurement of observer agreement for categorical data. Biometrics 33:159174

17. Dorgelo J, Willems TP, Geluk CA, van Ooijen PM, Zijlstra F, Oudkerk M (2005) Multidetector computed tomographyguided treatment strategy in patients with non-ST elevation acute coronary syndromes: a pilot study. Eur Radiol 15:708713

18. Mühlenbruch G, Seyfarth T, Soo CS, Pregalathan N, Mahnken AH (2007) Diagnostic value of 64-slice multidetector row cardiac CTA in symptomatic patients. Eur Radiol 17:603-609

19. Raff GL, Gallagher MJ, O’Neill WW, Goldstein JA (2005) Diagnostic accuracy of noninvasive coronary angiography using 64-slice spiral computed tomography. J Am Coll Cardiol 46:552-557

20. Achenbach S (2006) Computed tomography coronary angiography. J Am Coll Cardiol 48:1919-1928

21. Chow BJ, Hoffmann U, Nieman K (2005) Computed tomographic coronary angiography: an alternative to invasive coronary angiography. Can J Cardiol 21:933-940

22. Fuster V (1995) Elucidation of the role of plaque instability and rupture in acute coronary events. Am J Cardiol 76:24C-33C

23. Davies MJ, Thomas A (1984) Thrombosis and acute coronary-artery lesions in sudden cardiac ischemic death. $\mathrm{N}$ Engl J Med 310:1137-1140

24. Qiao JH, Fishbein MC (1991) The severity of coronary atherosclerosis at sites of plaque rupture with occlusive thrombosis. J Am Coll Cardiol 17:1138-1142 